Relation between circuit current and plasma current in discharges with dielectric media

\title{
Relation between circuit current and plasma current density in plasma discharges with dielectric media
}

L. Giuliani, ${ }^{1,2}$ D. Grondona, ${ }^{1,2}$ and F. Minotti ${ }^{1,2, \text { a) }}$

1) Universidad de Buenos Aires, Facultad de Ciencias Exactas y Naturales, Departamento de Física, Buenos Aires, Argentina. C1428EGA

${ }^{2)}$ CONICET-Universidad de Buenos Aires, Instituto de Física del Plasma (INFIP), Buenos Aires,

Argentina. C1428EGA

(Dated: 16 April 2020)

The theoretical derivation of the relation between moving charges in the plasma and external circuit current was first established on a firm basis by Sato ${ }^{1}$ for the case of a discharge with constant applied voltage between bare electrodes. The generalization to time varying applied voltages was established by Morrow and $\mathrm{Sato}^{2}$, still considering bare electrodes. In the present work we extend the latter derivation to include general devices with arbitrarily arranged linear dielectric media, and multiple electrodes with time dependent voltages, of interest, for instance, for a Dielectric Barrier Discharge (DBD). In particular, the present derivation determines a general, practical expression for the reactive term present in Morrow and Sato formula. Using the expression derived we show how in simple DBD geometries usual circuit approximations of the plasma discharge can be obtained and its validity justified. Also, we consider the determination of the internal plasma current from the measurement of the external circuit current for a cylindrical DBD operated with helium at atmospheric pressure. Finally, we apply the theory to a three-electrode device in order to relate electric measurements to volume integrals on the plasma that were shown to be useful in order to quantify species production by electron impact.

\section{INTRODUCTION}

The question of what currents are induced in the external circuit of a plasma device by the motion of charged particles in the plasma was satisfactorily answered in the work of Sato ${ }^{1}$ (see this reference for a discussion of previous approaches). In that reference, for the case of a DC discharge with bare electrodes, the conservation of energy, together with a clever decomposition of the electric field into a laplacian and a Poissonlike component, allowed the determination of the circuit current in terms of a volume integral involving the density and velocity of charged species in the plasma. Later, Morrow and Sato $^{2}$ showed how the previous formulation could be extended to include the case of time varying discharges, still considering bare electrodes, and also including in the integral the contribution of the diffusion of charged particles.

In the present work we extend the derivations in $^{2}$ to include the presence of linear dielectric media, of importance, for instance, for the applications to a Dielectric Barrier Discharge (DBD). Since some devices that include dielectric media also use more than two electrodes ${ }^{3}$, we also consider the presence of multiple external power sources. Moreover, since a description in terms of charged species densities, velocities and diffusion processes enters the formalism only through their determining the total conduction current density, the derivations are done directly in terms of this current, and only for comparison purposes are charged species explicitly mentioned.

We find that the expression of Morrow and Sato is valid also when linear dielectric media are included. In addition, we derive a practical expression for the reactive contribution in their formula, valid for arbitrary geometries.

a)Electronic mail: minotti@df.uba.ar
We note that the validity of Morrow and Sato's expression in the case of devices with linear dielectric media is not an obvious result, since polarization of the dielectrics contributes to the energy content and generates polarization currents too. Also, conduction currents in the plasma lead to electric charge deposited on the dielectrics, which in turn influence the value of the charge in the electrodes.

The obtained expression relates circuit currents and voltages to an integral, over the volume of the plasma, of the scalar product of the conduction current density times the laplacian electric field. In this way, an immediate important application of the expression derived is the determination of the expected circuit current from a numerical simulation of a plasma discharge, since in that case the plasma current density can be determined.

For alternative applications further elaborations are necessary in order to obtain useful information, so that as an example we derive, for the case of DBDs with simple geometries, the relation between plasma current and measured circuit current and voltage, that is usually obtained from circuit models. In these approximations the plasma is modeled as a parallel circuit between a variable resistor and a fixed capacitor ${ }^{4,5}$. In deriving the plasma current from first principles, the validity of the approximations are thus clarified. Also, the obtained expression for a cylindrical DBD is used in an experiment with helium at atmospheric pressure to show how important characteristics of the plasma current are thus made apparent.

As a final example the formalism is applied to a particular three-electrode discharge that combines a DBD with a remote third electrode, at fixed high voltage, that favors the propagation of streamers across a relatively wide gap. In this case the formula is useful to estimate the importance of different regions of the plasma in the production of reactive species.

In section II we derive the general expression of the relation between plasma and circuit currents, together with the condi- 
tions for its application.

The derivation of simplified expressions from the general relation, valid for DBDs with simple geometries, usually obtained from circuit models of the plasma, is done in section III, together with an application to an actual experiment.

In section IV the formalism is applied to a three-electrode device, and elaborated in order to obtain expressions useful for the determination of active species production by electron impact.

Finally, some conclusions are made in section V.

\section{THEORETICAL DERIVATION}

For the electrical discharges in gases as those considered by Morrow and Sato the contribution of the magnetic field energy to the energy balance can be neglected. To obtain a quantitative estimation of the conditions for this to hold, we consider the device to have a characteristic length $L$, and operated with power sources of characteristic frequency $T^{-1}$. From Ampère-Maxwell equation it is thus readily seen that if the displacement current contributes significantly to the determination of the magnetic field, the ratio of magnetic energy to electric energy can be estimated as $\left(\varepsilon_{0}\right.$ and $\mu_{0}$ are the vacuum permittivity and permeability, and $c$ the speed of light in vacuum)

$$
\frac{U_{B}}{U_{E}}=\frac{B^{2}}{\mu_{0} \varepsilon_{0} E^{2}} \sim \frac{L^{2}}{c^{2} T^{2}},
$$

so that the well known condition for quasi-stationary regime,

$$
L^{2} \ll c^{2} T^{2},
$$

is a first one to be satisfied. Note that this condition ensures the relative smallness of the magnetic energy, in the quasistationary regime, even when the displacement current is important, as, for instance, in the operation of a capacitive device.

On the other hand, the magnitude $E_{C}$ of the conservative component of the electric field can be estimated through the voltage difference $V$ across the length $L$ as

$$
E_{C} \sim V / L,
$$

while the magnitude $E_{N C}$ of the non-conservative component can be estimated using Faraday equation as

$$
E_{N C} \sim \frac{L}{T} B
$$

In the quasi-stationary regime, using Ampère-Maxwell equation, the magnitude of the magnetic field can be related to the magnitude of the current $I$ flowing into the device as

$$
B \sim \mu_{0} I / L .
$$

This is so even if the displacement current is important, because in such a case this current and $I$ have similar magnitudes. The ratio of non-conservative to conservative components of the electric field can thus be estimated as

$$
\frac{E_{N C}}{E_{C}} \sim \frac{\mu_{0} I}{V} \frac{L}{T}=\frac{\mu_{0} c I}{V} \frac{L}{c T},
$$

while the ratio of magnetic energy to electric energy, considering as the magnitude of the electric field that of the conservative component, is

$$
\frac{U_{B}}{U_{E}} \sim \frac{B^{2}}{\mu_{0} \varepsilon_{0} E_{C}^{2}} \sim \frac{\mu_{0} I^{2}}{\varepsilon_{0} V^{2}}=\left(\frac{\mu_{0} c I}{V}\right)^{2} .
$$

Taking into account condition (1), so that $L<c T$, from relation (2) it is seen that the non-conservative component is small, relative to the conservative one, if the right-hand side of relation (3) is small as compared with unity, so that the only additional condition to (1), in order to be able to neglect magnetic effects in the description of the discharge is

$$
\left(\frac{\mu_{0} c I}{V}\right)^{2} \ll 1
$$

In the SI units used $\left(\mu_{0} c\right)^{2} \simeq 1.4 \times 10^{5} \mathrm{~V}^{2} \mathrm{~A}^{-2}$, so that, for instance, in a typical DBD with $I \simeq 10^{-1} \mathrm{~A}, V \simeq 1 \mathrm{kV}$, the magnetic energy is about three orders of magnitude smaller than the electric energy.

In this way, when conditions (1) and (4) are satisfied, the conservation of energy in devices that include multiple powered electrodes and arbitrarily arranged linear dielectric media can be written as ${ }^{6}$

$$
\begin{aligned}
\sum_{k} i_{k} V_{k} & =\int \mathbf{j} \cdot \mathbf{E} d \Omega+\frac{d}{d t} \frac{1}{2} \int \mathbf{E} \cdot \mathbf{D} d \Omega \\
& =\int \mathbf{j} \cdot \mathbf{E} d \Omega+\int \mathbf{E} \cdot \frac{\partial \mathbf{D}}{\partial t} d \Omega .
\end{aligned}
$$

where $i_{k}$ is the discharge current that flows to the electrode $k$, with instantaneous voltage $V_{k}$ (all voltages are referred to ground, or zero voltage), $\mathbf{j}$ is the conduction current density in the plasma, and $\mathbf{E}$ and $\mathbf{D}$ the electric and displacement fields, respectively. The volume integrals are formally extended to all space. Taking advantage of the linearity of the equations for given charge distributions and voltages, the fields can be decomposed into a Laplace component $\mathbf{E}_{L}$, corresponding to the electric field generated by the biased electrodes, but without free electric charge present, and a Poisson component $\mathbf{E}_{P}$, due to the free charge present, of volume density $\rho$, and considering grounded electrodes. These fields thus satisfy the equations

$$
\begin{aligned}
\mathbf{E}_{L, P} & =-\nabla \phi_{L, P}, \\
\nabla \cdot \mathbf{D}_{L} & =0, \\
\nabla \cdot \mathbf{D}_{P} & =\rho,
\end{aligned}
$$

with the relation

$$
\mathbf{D}_{L, P}=\Xi \cdot \mathbf{E}_{L, P},
$$

where $\Xi$ is the dielectric tensor, which is symmetric: $\Xi^{T}=\Xi$, with $T$ denoting tensor transposition ${ }^{7}$. The dielectric tensor has in general a dependence on the space position, so as to model any complex arrangement of different dielectrics, including gases and vacuum. Use of a general, not necessarily isotropic, linear medium does not complicate the derivations 
to follow, while allowing to extend the range of possible applications.

The boundary conditions are that the potentials $\phi_{L, P}$ decay to zero at infinity, and that at the $k$ electrode, with instantaneous voltage $V_{k}$, they satisfy

$$
\begin{aligned}
& \left.\phi_{L}\right|_{k}=V_{k}, \\
& \left.\phi_{P}\right|_{k}=0 .
\end{aligned}
$$

Expression (5) can thus be split as

$$
\begin{aligned}
\sum_{k} i_{k} V_{k}= & \int \mathbf{j} \cdot \mathbf{E}_{L} d \Omega+\int \mathbf{j} \cdot \mathbf{E}_{P} d \Omega+\int \mathbf{E}_{L} \cdot \frac{\partial \mathbf{D}}{\partial t} d \Omega \\
& +\int \mathbf{E}_{P} \cdot \frac{\partial \mathbf{D}_{P}}{\partial t} d \Omega+\int \mathbf{E}_{P} \cdot \frac{\partial \mathbf{D}_{L}}{\partial t} d \Omega .
\end{aligned}
$$

We note that in the problem of determining the Poisson part of the field all boundaries have zero potential, so that no external power is supplied to this component. The conservation of energy for the Poisson field alone must thus satisfy

$$
\int \mathbf{j} \cdot \mathbf{E}_{P} d \Omega+\int \mathbf{E}_{P} \cdot \frac{\partial \mathbf{D}_{P}}{\partial t} d \Omega=0
$$

The proof of (7) can be obtained directly from the fundamental equations noting that

$$
\begin{aligned}
\int \mathbf{E}_{P} \cdot \frac{\partial \mathbf{D}_{P}}{\partial t} d \Omega & =-\int \nabla \phi_{P} \cdot \frac{\partial \mathbf{D}_{P}}{\partial t} d \Omega \\
& =-\int \nabla \cdot\left(\phi_{P} \frac{\partial \mathbf{D}_{P}}{\partial t}\right) d \Omega+\int \phi_{P} \frac{\partial}{\partial t} \nabla \cdot \mathbf{D}_{P} d \Omega
\end{aligned}
$$

Since $\phi_{P}=0$ on all boundaries, use of Gauss theorem immediately shows that the first integral in the second line is zero. Using also Gauss law: $\nabla \cdot \mathbf{D}_{P}=\rho$, and charge conservation: $\partial \rho / \partial t=-\nabla \cdot \mathbf{j}$, one obtains the desired result as

$$
\begin{aligned}
\int \mathbf{E}_{P} \cdot \frac{\partial \mathbf{D}_{P}}{\partial t} d \Omega & =\int \phi_{P} \frac{\partial}{\partial t} \nabla \cdot \mathbf{D}_{P} d \Omega=\int \phi_{P} \frac{\partial \rho}{\partial t} d \Omega \\
& =-\int \phi_{P} \nabla \cdot \mathbf{j} d \Omega \\
& =-\int \nabla \cdot\left(\phi_{P} \mathbf{j}\right) d \Omega+\int \mathbf{j} \cdot \nabla \phi_{P} d \Omega \\
& =-\int \mathbf{j} \cdot \mathbf{E}_{P} d \Omega,
\end{aligned}
$$

where Gauss theorem and the condition $\phi_{P}=0$ on all boundaries were used.

The last integral in (6) is readily seen to be zero too:

$$
\begin{aligned}
\int \mathbf{E}_{P} \cdot \frac{\partial \mathbf{D}_{L}}{\partial t} d \Omega & =-\int \nabla \phi_{P} \cdot \frac{\partial \mathbf{D}_{L}}{\partial t} d \Omega \\
& =-\int \nabla \cdot\left(\phi_{P} \frac{\partial \mathbf{D}_{L}}{\partial t}\right) d \Omega+\int \phi_{P} \frac{\partial}{\partial t}\left(\nabla \cdot \mathbf{D}_{L}\right) d \Omega \\
& =0 .
\end{aligned}
$$

In the last line $\phi_{P}=0$ on all boundaries and Gauss theorem were again used, together with $\nabla \cdot \mathbf{D}_{L}=0$.

Relation (5) thus reduces to

$$
\sum_{k} i_{k} V_{k}=\int \mathbf{j} \cdot \mathbf{E}_{L} d \Omega+\int \mathbf{E}_{L} \cdot \frac{\partial \mathbf{D}}{\partial t} d \Omega
$$

We consider now the last integral in (8)

$$
\begin{aligned}
\int \mathbf{E}_{L} \cdot \frac{\partial \mathbf{D}}{\partial t} d \Omega & =\int \mathbf{E}_{L} \cdot\left(\Xi \cdot \frac{\partial \mathbf{E}}{\partial t}\right) d \Omega \\
& =\int\left(\Xi^{T} \cdot \mathbf{E}_{L}\right) \cdot \frac{\partial \mathbf{E}}{\partial t} d \Omega=-\int \mathbf{D}_{L} \cdot \nabla \frac{\partial \phi}{\partial t} d \Omega \\
& =-\int \nabla \cdot\left(\mathbf{D}_{L} \frac{\partial \phi}{\partial t}\right) d \Omega+\int \frac{\partial \phi}{\partial t} \nabla \cdot \mathbf{D}_{L} d \Omega \\
& =-\int \frac{\partial \phi}{\partial t} \mathbf{D}_{L} \cdot \mathbf{n} d S,
\end{aligned}
$$

where $\phi=\phi_{L}+\phi_{P}$. The symmetry of the dielectric tensor was used in the second line, and also that $\nabla \cdot \mathbf{D}_{L}=0$ in the last line. The integrals in this last line are extended to all boundaries: formally infinity and the boundary of the electrodes.

The boundaries at infinity do not contribute to the integral since the involved products of fields and potentials due to localized charges decay to zero sufficiently rapidly.

In case that the boundary corresponds to that of the $k$ electrode one has $\mathbf{D}_{L} \cdot \mathbf{n}=-\sigma_{k}^{L}$, with $\sigma_{k}^{L}$ the local Laplace charge surface density on the $k$ conductor (that induced by the biasing of all the electrodes, without free electric charges present outside the electrodes). Also, at that boundary, $\partial \phi / \partial t=d V_{k} / d t$. In this way

$$
\int \mathbf{E}_{L} \cdot \frac{\partial \mathbf{D}}{\partial t} d \Omega=\sum_{k} \frac{d V_{k}}{d t} \int \sigma_{k}^{L} d S=\sum_{k} \frac{d V_{k}}{d t} Q_{k}^{L},
$$

where $Q_{k}^{L}$ is the total Laplace charge of the electrode $k$.

The charge $Q_{k}^{L}$ can be determined using the capacitance coefficients $C_{k n}$, evaluated (or measured) for the device without plasma present, as

$$
Q_{k}^{L}=\sum_{n} C_{k n} V_{n}
$$

One can thus finally write

$$
\sum_{k} i_{k} V_{k}=\int \mathbf{j} \cdot \mathbf{E}_{L} d \Omega+\sum_{k, n} C_{k n} V_{n} \frac{d V_{k}}{d t} .
$$

If just two electrodes are present, with relative voltage $V(t)$, expression (9) reduces to

$$
i=\frac{1}{V} \int \mathbf{j} \cdot \mathbf{E}_{L} d \Omega+C \frac{d V}{d t},
$$

where $C$ is the device capacitance. In this way, the result of Morrow and $\mathrm{Sato}^{2}$ is reobtained if the current density in Eq. (10) is expressed in terms of the charge carriers densities and drift velocities:

$$
\mathbf{j}=\sum_{i} q_{i}\left(N_{i} \mathbf{w}_{i}-D_{i} \nabla N_{i}\right),
$$

where $q_{i}$ is the charge of the species $i, N_{i}$ its number density, $\mathbf{w}_{i}$ the species drift velocity, and $D_{i}$ its diffusion coefficient.

Expression (10) has the practical advantage, over the corresponding Eq. (23) $\mathrm{in}^{2}$, of expressing the inductive power term, the last one in both expressions, in terms of the capacitance and time derivative of the voltage. A similar term was obtained in that reference, but restricted to a particular device with plane, bare parallel electrodes, and neglecting fringe field effects. 


\section{APPLICATION TO SIMPLE DBD GEOMETRIES AND RELATION TO CIRCUIT MODELS OF THE DISCHARGE}

Usual designs of DBD devices have a high degree of symmetry, with plane or cylindrical electrodes. In those cases, if border effects can be neglected, the laplacian field $\mathbf{E}_{L}$ has just one vector component that depends only on the coordinate corresponding to that component, which we denote generically as $\xi$. In this way, one can write the integral in Eq. (10) as

$$
\begin{aligned}
\int \mathbf{j} \cdot \mathbf{E}_{L} d \Omega & =\int d \xi E_{L}(\xi) \int_{S(\xi)} j \xi d S \\
& =\int d \xi E_{L}(\xi) I(\xi)
\end{aligned}
$$

where $E_{L}$ and $j_{\xi}$ are the $\xi$ components of the corresponding vectors, $S(\xi)$ is the surface corresponding to a constant $\xi$ in the gap, and $I(\xi)$ is the instantaneous current crossing that surface.

If the charge conservation equation

$$
\frac{\partial \rho}{\partial t}+\nabla \cdot \mathbf{j}=0
$$

is integrated over a given surface $S(\xi)$, one readily obtains

$$
\frac{\partial \Sigma}{\partial t}+\frac{\partial I}{\partial \xi}=0
$$

where $\Sigma$ is given by

$$
\Sigma(\xi, t)=\int_{S(\xi)} \rho d S
$$

so that $\Sigma(\xi, t) d \xi$ is the electric charge between the surfaces $S(\xi)$ and $S(\xi+d \xi)$.

Considering signals with time resolution below the transit time of ionization fronts and/or streamers across the gap (which is smaller than about 10ns for millimeter sized gaps ${ }^{8}$ ), charge variation manifests only in very thin layers at the dielectric surfaces where charge is deposited, so that the current $I$ is spatially uniform, except in those layers, thus allowing to approximate Eq. (11) as

$$
\int \mathbf{j} \cdot \mathbf{E}_{L} d \Omega=I \int E_{L}(\xi) d \xi .
$$

Noting further that the laplacian field can be written as the instantaneous voltage difference $V$ between the electrodes, times a factor $f(\xi)$ depending only on the geometry and physical characteristics of the device, Eq. (10) can be reduced to

$$
i=I \int f(\xi) d \xi+C \frac{d V}{d t}
$$

where the integration extends to the length of the gap.

As examples we consider the devices shown in Fig (1). The first device has plane electrodes of area $A$, each covered with dielectrics of width $d_{1}$ and $d_{2}$, and permittivities $\varepsilon_{1}$ and $\varepsilon_{2}$, respectively. The gap between the dielectrics has a width $d_{G}$.
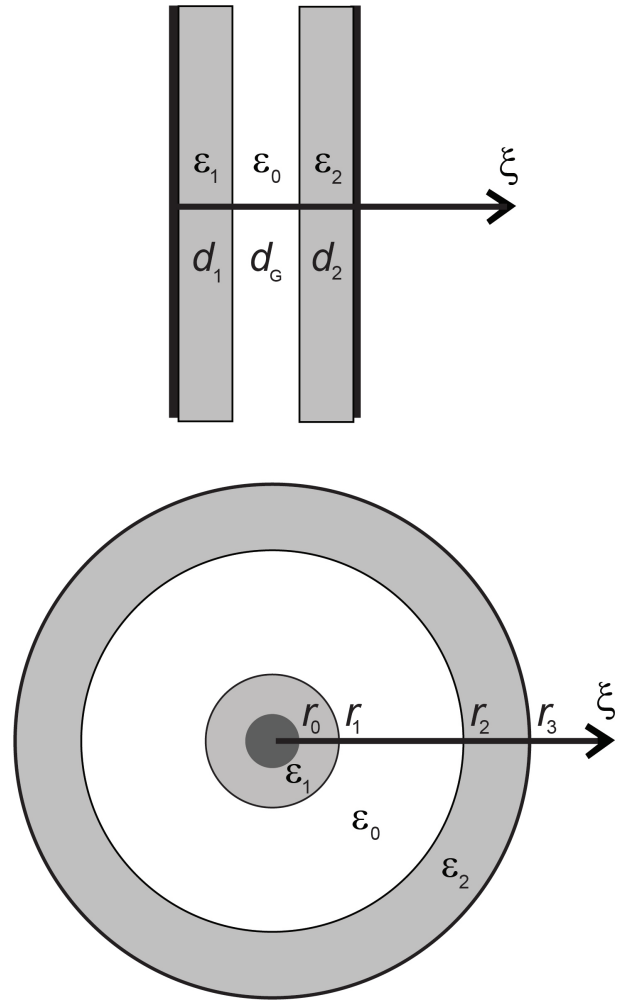

FIG. 1. Plane and cylindrical DBD devices.

Neglecting fringe fields the corresponding capacitance is

$$
C=\frac{A}{d_{1} / \varepsilon_{1}+d_{G} / \varepsilon_{0}+d_{2} / \varepsilon_{2}},
$$

with $\varepsilon_{0}$ the vacuum permittivity. The laplacian electric field in the gap has $\xi$ component

$$
E_{L}=\frac{V}{\varepsilon_{0} d_{1} / \varepsilon_{1}+d_{G}+\varepsilon_{0} d_{2} / \varepsilon_{2}},
$$

so that

$$
\int f(\xi) d \xi=\frac{d_{G}}{\varepsilon_{0} d_{1} / \varepsilon_{1}+d_{G}+\varepsilon_{0} d_{2} / \varepsilon_{2}}=\frac{C d_{G}}{\varepsilon_{0} A} .
$$

One thus obtains the expression of the plasma current as

$$
I=\frac{\varepsilon_{0} A}{C d_{G}}\left(i-C \frac{d V}{d t}\right)
$$

The second device has cylindrical electrodes, the inner one of radius $r_{0}$ and the outer one of internal radius $r_{3}$. A dielectric 
of permittivity $\varepsilon_{1}$ covers the inner electrode up to radius $r_{1}$, and a second dielectric of permittivity $\varepsilon_{2}$ occupies the region between radius $r_{2}$ and $r_{3}$, the gap is in between radius $r_{1}$ and $r_{2}$.

The device capacitance is given by

$$
C=\frac{2 \pi L \varepsilon_{0}}{\frac{\varepsilon_{0}}{\varepsilon_{1}} \ln \left(\frac{r_{1}}{r_{0}}\right)+\ln \left(\frac{r_{2}}{r_{1}}\right)+\frac{\varepsilon_{0}}{\varepsilon_{2}} \ln \left(\frac{r_{3}}{r_{2}}\right)},
$$

where $L$ is the device axial length. The expression of the $\xi$ (radial) component of $\mathbf{E}_{L}$ is

$$
E_{L}=\frac{V / \xi}{\frac{\varepsilon_{0}}{\varepsilon_{1}} \ln \left(\frac{r_{1}}{r_{0}}\right)+\ln \left(\frac{r_{2}}{r_{1}}\right)+\frac{\varepsilon_{0}}{\varepsilon_{2}} \ln \left(\frac{r_{3}}{r_{2}}\right)}
$$

so that

$$
\begin{aligned}
\int f(\xi) d \xi & =\frac{\ln \left(\frac{r_{2}}{r_{1}}\right)}{\frac{\varepsilon_{0}}{\varepsilon_{1}} \ln \left(\frac{r_{1}}{r_{0}}\right)+\ln \left(\frac{r_{2}}{r_{1}}\right)+\frac{\varepsilon_{0}}{\varepsilon_{2}} \ln \left(\frac{r_{3}}{r_{2}}\right)} \\
& =\frac{C}{2 \pi L \varepsilon_{0}} \ln \left(\frac{r_{2}}{r_{1}}\right) .
\end{aligned}
$$

The plasma current can thus be related to the measured voltage and circuit current as

$$
I=\frac{2 \pi L \varepsilon_{0}}{C \ln \left(r_{2} / r_{1}\right)}\left(i-C \frac{d V}{d t}\right) .
$$

Relations (14) and (15) correspond to those usually obtained using circuit models of the plasma ${ }^{4,5}$. The derivations shown here thus help to clarify the conditions for the validity of those expressions.

An interesting point is that the factor multiplying $i-$ $C d V / d t$ in Eqs. (14) and (15) is larger than one (this factor is equal to one for the case of bare electrodes.) Polarization currents in the dielectric media oppose the current in the plasma, reducing the effect of the latter on the circuit current.

As an example we consider now the application of Eq. (15) to a cylindrical DBD operated with atmospheric pressure helium. The device used is like the second one shown in Fig. (1), and represented in side view in Fig. (2). The discharge is made between two coaxial electrodes covered by dielectric barriers. The inner electrode consists in an iron rod of $3 \mathrm{~mm}$ diameter covered by a glass capillary tube sealed at the tip, with an external diameter of $6 \mathrm{~mm}$. The $23 \mathrm{~mm}$ long outer electrode is an aluminum tape attached to an acrylic tube of $1.5 \mathrm{~mm}$ thickness and an external diameter of $10 \mathrm{~mm}$.

The central electrode was connected to a high voltage power source $(0-25 \mathrm{kV}, 10 \mathrm{kHz})$ and its voltage $V$ measured using a high voltage probe (Tektronix P6015A 1000X/3.0 $\mathrm{pf} / 100 \mathrm{MHz}$ ). The external electrode was either grounded through a $50 \Omega$ resistor, to measure the discharge current, or through a $10 \mathrm{nF}$ capacitor to measure the charge $Q$ transported to the external electrode.

By measuring the charge $Q$ as a function of the applied voltage $V$ for a sufficiently low voltage amplitude, in order to avoid gas breakdown, the device capacitance was measured, resulting in $C=(4.34 \pm 0.02) \mathrm{pF}$.

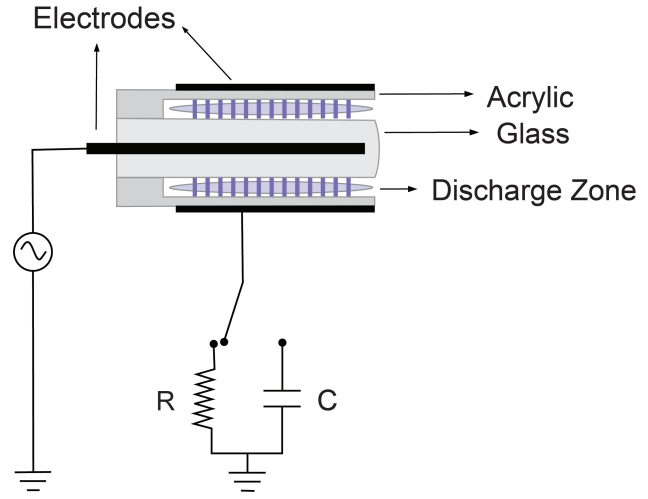

FIG. 2. Experimental device.

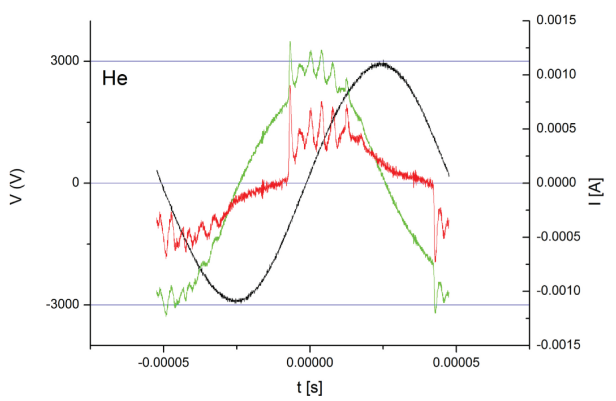

FIG. 3. Applied voltage (black), circuit current $i$ (green) and corresponding plasma current $I$ (red), for a cylindrical DBD with He as operating gas. The amplitude of the voltage is about $3 \mathrm{kV}$. The circuit current has a reactive component of amplitude about $1 \mathrm{~mA}$, with peaks of approximately $0.25 \mathrm{~mA}$. The plasma current presents peaks of about $0.4 \mathrm{~mA}$, above a mean component of similar magnitude that decays in about $20 \mu \mathrm{s}$.

In Fig. (3) we present the measured circuit current $i$ together with the applied voltage and the plasma current $I$ determined using Eq.( 15).

As discussed above, the relative factor between plasma and circuit currents, that in the right-hand side of Eq. (15), is larger than one, and indicates, for the considered device, that the plasma current is about $60 \%$ larger than the circuit current minus its purely reactive component.

Also, a slow decay to zero of the plasma current is clearly seen after the succession of current peaks, a feature that is not apparent in the circuit current. Moreover, the relative height between different peaks in the plasma current cannot be directly appreciated in the circuit current. 


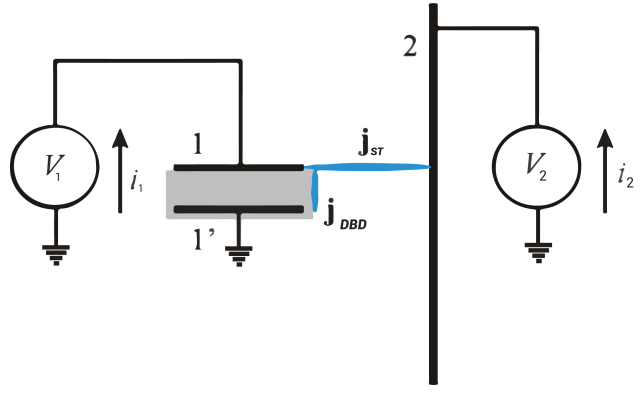

FIG. 4. Schematic of the three-electrode device. $V_{1}$ and $V_{2}$ are AC and DC voltage sources, respectively. Electrodes 1 and $1^{\prime}$ correspond to the DBD, with electrode $1^{\prime}$ covered with dielectric medium. Electrodes 1 and 2 are bare.

\section{APPLICATION TO A THREE-ELECTRODE DEVICE}

We consider now the application to a device designed for polluted gas remediation that combines a DBD with a third electrode that favors the development of streamers across a relatively wide gap ${ }^{3}$. A schematic of the device is shown in Fig. (4) including the definition of the currents and voltages employed.

Eq. (9) applied to this device is written as

$$
\begin{aligned}
i_{1} V_{1}+i_{2} V_{2}= & \int \mathbf{j}_{D B D} \cdot \mathbf{E}_{L} d \Omega+\int \mathbf{j}_{S T} \cdot \mathbf{E}_{L} d \Omega \\
& +\left(C_{11} V_{1}+C_{12} V_{2}\right) \frac{d V_{1}}{d t},
\end{aligned}
$$

where the plasma density current was explicitly split into the contribution from the DBD current, $\mathbf{j}_{D B D}$, and that from the streamers, $\mathbf{j}_{S T}$. Also, it was taken into account that the voltage $V_{2}$ is constant.

The density currents $\mathbf{j}_{D B D}$ and $\mathbf{j}_{S T}$ amount to currents $I_{D B D}$ and $I_{S T}$ leaving (when they are positive) the bare electrode of the DBD arrangement, respectively, so that the charge $Q_{1}$ on that electrode satisfies

$$
\frac{d Q_{1}}{d t}=C_{11} \frac{d V_{1}}{d t}=i_{1}-I_{D B D}-I_{S T}
$$

A similar relation can be applied to the bare electrode 2, which collects the current $I_{S T}$, to give

$$
\frac{d Q_{2}}{d t}=C_{12} \frac{d V_{1}}{d t}=i_{2}+I_{S T}
$$

From the last two equations one obtains

$$
\begin{aligned}
I_{D B D} & =i_{1}+i_{2}-\left(C_{11}+C_{12}\right) \frac{d V_{1}}{d t}, \\
I_{S T} & =C_{12} \frac{d V_{1}}{d t}-i_{2} .
\end{aligned}
$$

As observed in the experiment, and expected from the conditions for streamer propagation ${ }^{9}$, the current density $\mathbf{j}_{S T}$ is distributed in a very localized axial position, in the radial extension between the bare electrode of the DBD and the electrode 2. In this region the laplacian field at fixed axial position is an axially symmetric function dependent on radius, so that, with the same considerations used in the simplified circuit models, one can write

$$
\begin{aligned}
\int \mathbf{j}_{S T} \cdot \mathbf{E}_{L} d \Omega & =I_{S T} \int_{r_{1}}^{r_{2}} E_{L} d r=-I_{S T}\left(V_{2}-V_{1}\right) \\
& =\left(i_{2}-C_{12} \frac{d V_{1}}{d t}\right)\left(V_{2}-V_{1}\right),
\end{aligned}
$$

where $E_{L}$ denotes the radial component of $\mathbf{E}_{L}$, and Eq. (18) was used in the second line. This relation, together with Eq. (16), thus allows us to write

$$
\int \mathbf{j}_{D B D} \cdot \mathbf{E}_{L} d \Omega=\left(i_{1}+i_{2}\right) V_{1}-\left(C_{11}+C_{12}\right) V_{1} \frac{d V_{1}}{d t} .
$$

As done $i^{3}$ integrals as those in the left-hand sides of Eqs. (19) and (20) can be seen to be proportional (through a coefficient dependent on the particular reaction involved) to the production of species by electron impact. In this way, one can relate this species production to the electric measurements on the device, and also to discriminate the role of the different regions of the discharge in this production.

\section{CONCLUSIONS}

Extending the derivations by Sato ${ }^{1}$ and Morrow and Sato ${ }^{2}$ we have obtained from first principles the relation between internal plasma current density and circuit current, in electrical discharges in devices containing linear dielectric media.

The expression derived is particularly useful in numerical simulations in order to relate the simulated plasma current to the expected circuit current in an actual experiment.

The relation obtained by Morrow and Sato is shown to be valid also when dielectrics are included, if the reactive term in their formula is conveniently expressed in terms of the actual device capacitance.

More phenomenological approaches, that treat the plasma in a DBD as a circuit with a variable resistor in parallel with a fixed capacitor, are also shown to be valid in usual simple geometries. The conditions are that end effects can be neglected, and that relatively slowly varying electric signals are considered, that do not resolve the rapid evolution of ionization avalanches and/or streamer propagation.

As a direct application to an experiment, the plasma current in a cylindrical DBD operated in atmospheric pressure helium was obtained from the circuit signals, showing characteristics of the plasma current profile that are not apparent in the circuit current.

Finally, many DBD devices are intended for environmental and biological applications, in which the production of reactive species is of fundamental importance. As shown $\mathrm{in}^{3}$, the rate of production of species involving electron impact can be directly related to the volume integrals that appear in the formalism, so that we finally show its application to a particular three-electrode device designed for polluted gas remediation. 


\section{ACKNOWLEDGMENTS}

We wish to acknowledge the following institutional grants: grant 20020150100096BA from the University of Buenos Aires, grant GI 11220120100453 from the National Council of Scientific and Technical Research (CONICET), and grant 2010-0771 from the National Agency of Scientific and Technological Promotion (ANPCyT).

${ }^{1}$ N. Sato, Journal of Physics D: Applied Physics 134, L3 (1980).
${ }^{2}$ R. Morrow and N. Sato, Journal of Physics D: Applied Physics 32, L20 (1999).

${ }^{3}$ J. L. Gallego, D. Grondona, and F. Minotti, Journal of Physics D: Applied Physics 47, 205202 (2014).

${ }^{4}$ A. Pipa and R. Brandenburg, Atoms 7, 14 (2019).

5 J.-S. Boisvert, L. Stafford, N. Naudé, J. Margot, and F. Massines, Plasma Sources Science and Technology 27, 035005 (2018).

${ }^{6}$ J. D. Jackson, Classical Electrodynamics (John Wiley \& Sons, 1999)

${ }^{7}$ L. D. Landau and E. M. Lifshitz, Electrodynamics of Continuous Media (Pergamon Press, Oxford, 1984)

${ }^{8}$ Y. P. Raizer, Gas Discharge Physics (Springer, Berlin, 1991).

${ }^{9}$ J. L. Gallego, F. Minotti, and D. Grondona, IEEE Transactions on Plasma Science 45, 54 (2017) 

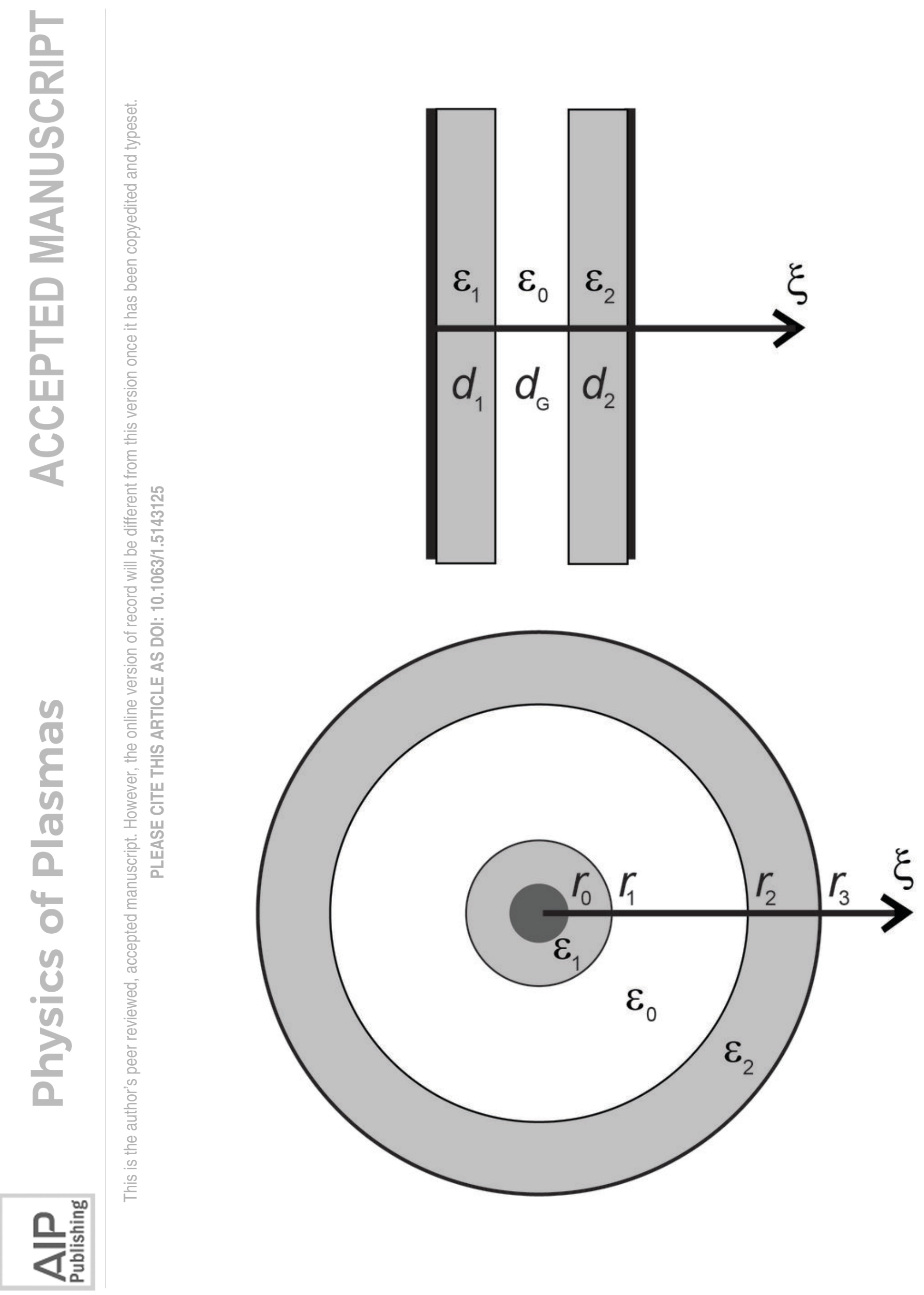


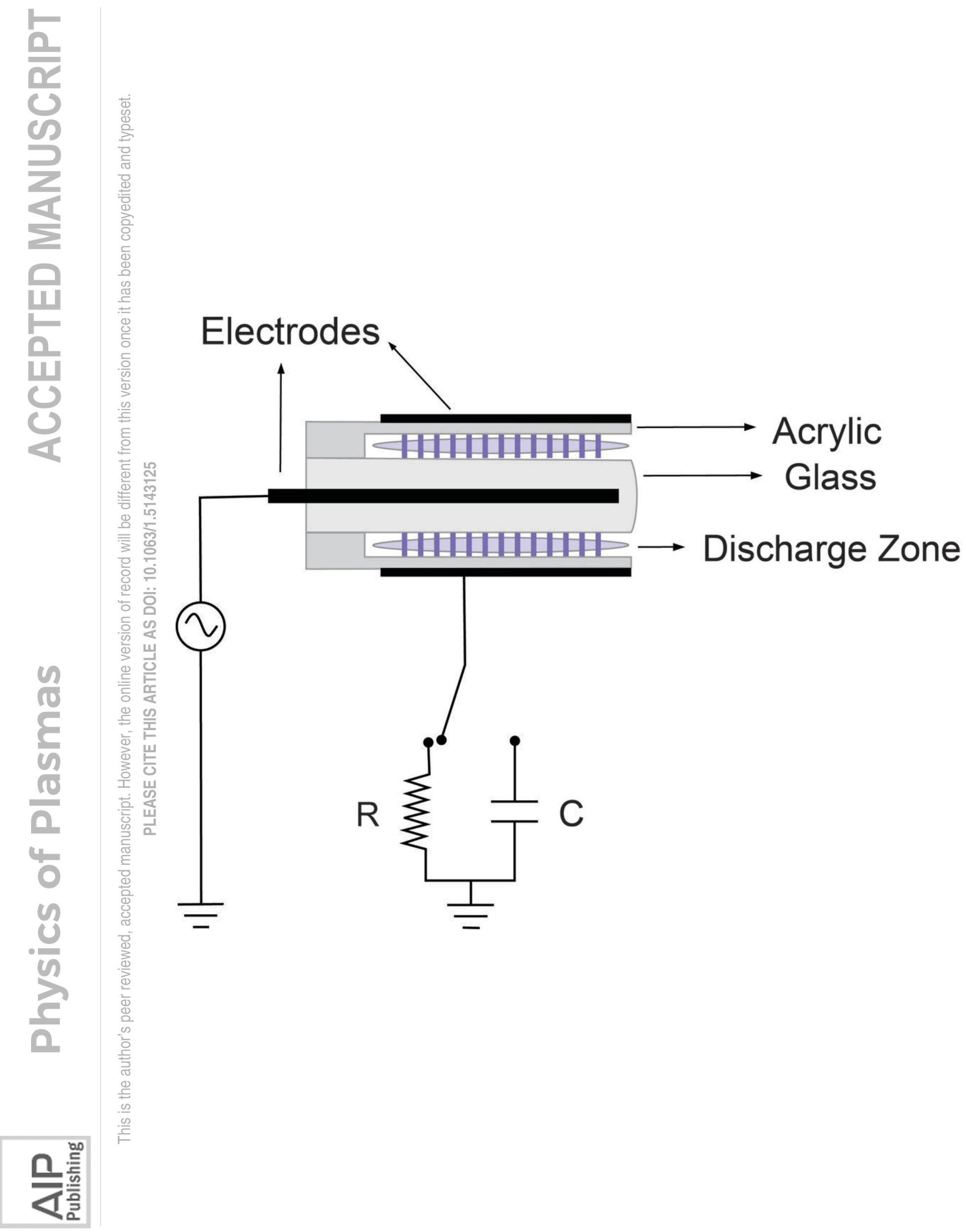




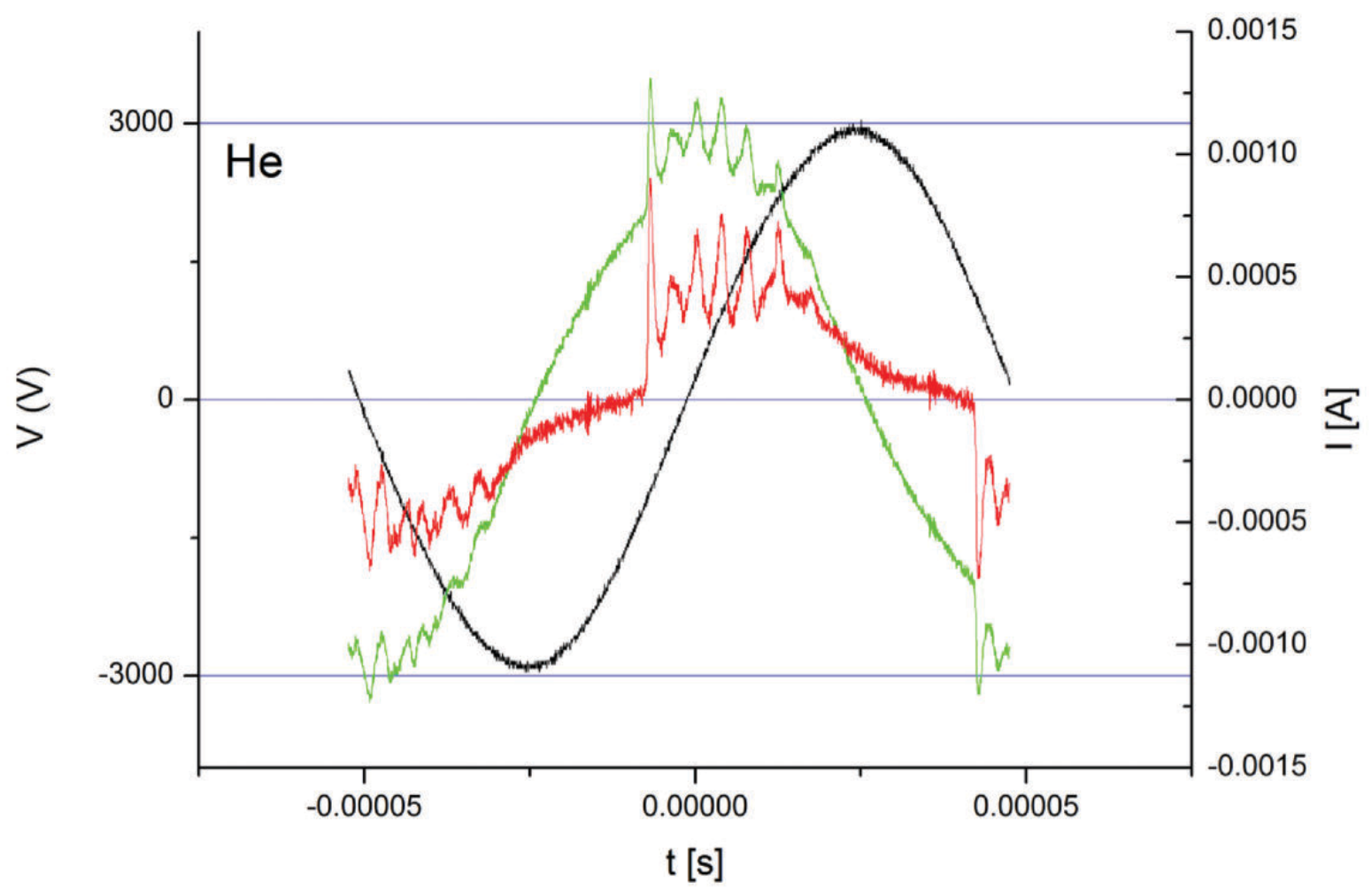




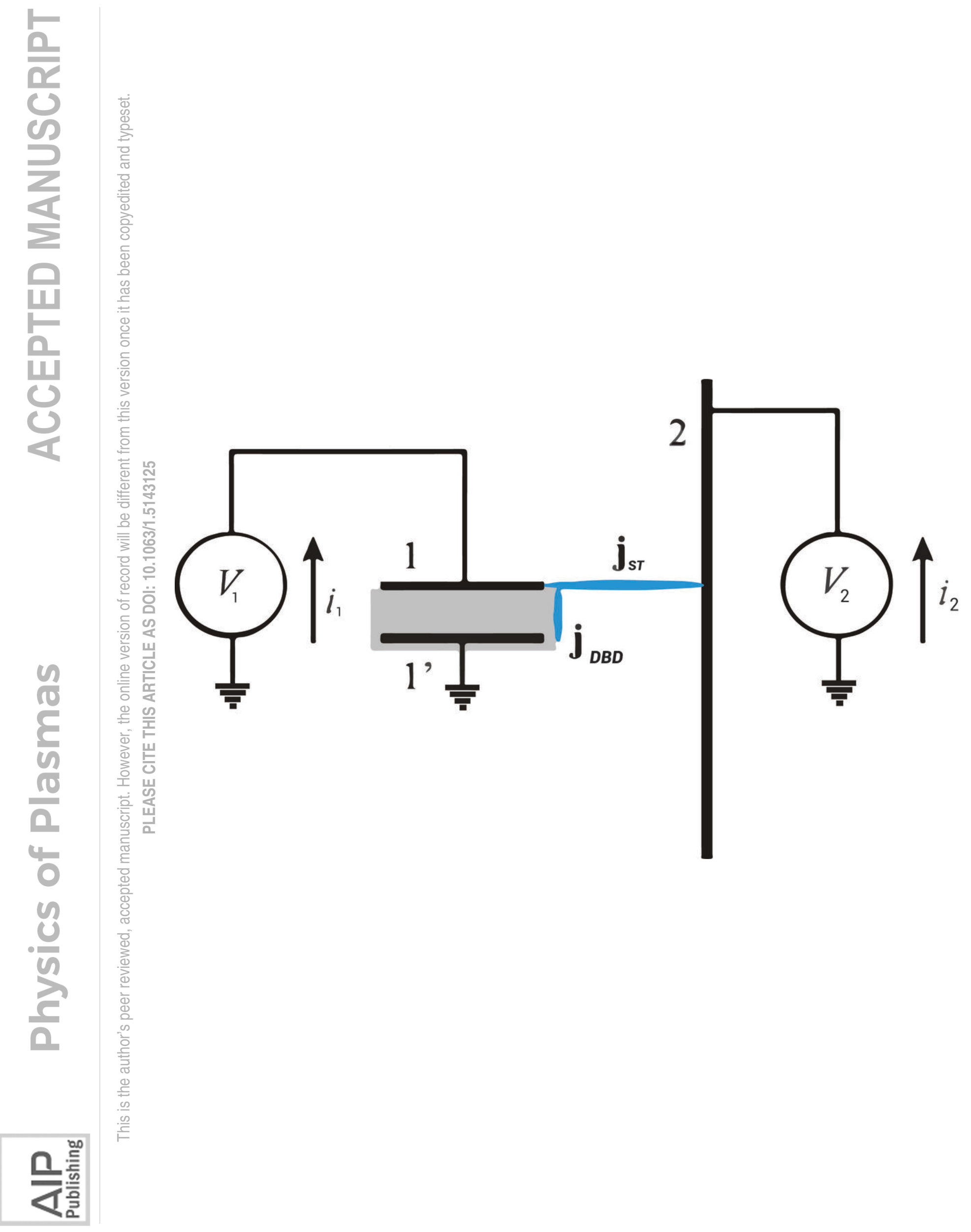

